\title{
Other Healthcare Practitioners and Technical Occupations
}

National Cancer Institute

\section{Source}

National Cancer Institute. Other Healthcare Practitioners and Technical Occupations. NCI Thesaurus. Code C122544.

Healthcare practitioners and technical occupations not specifically identified elsewhere. 